\title{
Assessing the prognostic scores for the prediction of the mortality of patients with acute-on-chronic liver failure: a retrospective study
}

\author{
Yue Zhang ${ }^{\text {Equal first author, 1 }}$, Yuan Nie ${ }^{\text {Equal first author, 1 }}$ Linxiang Liu $^{1}$, Xuan Zhu ${ }^{\text {Corresp. } 1}$ \\ ${ }^{1}$ Department of Gastroenterology, The First Affiliated Hospital of Nanchang University, Nanchang, Jiangxi, China \\ Corresponding Author: Xuan Zhu \\ Email address: waiyongtg@163.com
}

Background: Acute-on-chronic liver failure (ACLF), which is characterized by rapid deterioration of liver function and multiorgan failure, has high mortality. This study was designed to identify prognostic scores to predict short-term and long-term outcome in patients with ACLF to facilitate early treatment and thereby improve patient survival. Materials and Methods: We retrospectively analyzed 102 ACLF patients who were hospitalized in the gastroenterology department. The EASL-CLIF criteria were used to define the ACLF. The demographic characteristics and biochemical examination results of the patients were acquired, and seven scores (CTP score, MELD score, MELD-Na, CLIF ACLF score, CLIF-C OF score, CLIF SOFA score ) were calculated 24 hours after admission. All patients were observed until loss to follow-up, death, or specific follow-up times (28 days, 3 months , 6 months), which were calculated after the initial hospital admission. The receiver operating characteristic (ROC) curve was employed to estimate the power of six scores to forecast ACLF patients' outcome. Results: All scores were distinctly higher in nonsurviving patients than in surviving patients and had predictive value for outcome in patients with ACLF at all time points $(P<0.050)$. The areas under the ROC curve (AUROCs) of the CLIF-SOFA score were higher than those of other scores at all time points. The comparison of the AUROC of the CLIF-SOFA score with other scores was statistically significant at 28 days $(P<0.050)$, which was the only time point at which it was greater than 0.800. Conclusion: Patients with ACLF have high mortality. These six scores are effective tools for assessing the prognosis of ACLF patients. The CLIF-SOFA score is especially effective for evaluating 28-day mortality. 
1 Manuscript type: Original Article

2 Title: Assessing the prognostic scores for the prediction of the mortality of patients with acute-

3 on-chronic liver failure: a retrospective study

4 Running title: Assessing of prognostic score in ACLF patients

5 Authors: Yue Zhang ${ }^{1 \dagger}$, Yuan $\mathrm{Nie}^{1 \dagger}$, Linxiang Liu ${ }^{1}$, Xuan $\mathrm{Zhu}^{1}$

6 Abbreviated name: Zhang Y; Nie Y; Liu LX; Zhu X.

7 City: Nanchang, China

8 Author affiliations

9 1: Department of Gastroenterology, The First Affiliated Hospital of Nanchang University, 10 Nanchang,330006, China.

11 * Corresponding author

12 Xuan Zhu,

13 Department of Gastroenterology, The First Affiliated Hospital of Nanchang University No.17, 14 Yongwaizhengjie Road, Donghu District Nanchang 330006, Jiangxi, China.

15 (E-mail address: waiyongtg@163.com)

16 CRediT authorship contribution statement:

17 Yue Zhang :Writing - original draft.

18 Yuan Nie : Methodology, Writing - original draft.

Linxiang Liu:Data curation and Formal analysis.

Xuan Zhu: Conceptualization, Funding acquisition, Supervision, Writing - review \& editing.

Funding: This study was supported by the National Natural Science Foundation of China (grant number: 81660110) and the "Gan-Po Talent 555" Project of Jiangxi Province.

Ethical approval: This study was approved by the Ethics Committee of The First Affiliated Hospital of Nanchang University (No.2015-1203). Written informed consent was obtained from all participants.

Competing interest: No benefits in any form have been received or will be received from a commercial party related directly or indirectly to the subject of this article.

Acknowledgment: We would like to thanks the National Natural Science Foundation of China and the "Gan-Po Talent 555" Project of Jiangxi Province for the economic support. 


\title{
Assessing the prognostic scores for the prediction of the mortality of patients with
} acute-on-chronic liver failure: a retrospective study

\author{
Yue Zhang ${ }^{1 \dagger}$, Yuan $\mathrm{Nie}^{1 \dagger}$, Linxiang $\mathrm{Liu}^{1}$, Xuan $\mathrm{Zhu}^{1 *}$ \\ ${ }^{1}$ Department of Gastroenterology, The First Affiliated Hospital of Nanchang University, Nanchang, Jiangxi, \\ China \\ $\dagger \mathrm{YZ}$ and $\mathrm{YN}$ contributed equally to this study.
}

Correspondence to : Xuan Zhu ${ }^{1 *}$, Department of Gastroenterology, The First Affiliated Hospital of Nanchang University No.17, Yongwaizhengjie Road, Donghu District Nanchang 330006, Jiangxi, China; E-mail addresses: waiyongtg@163.com

\section{Abstract}

Background: Acute-on-chronic liver failure (ACLF), which is characterized by rapid deterioration of liver function and multiorgan failure, has high mortality. This study was designed to identify prognostic scores to predict short-term and long-term outcome in patients with ACLF to facilitate early treatment and thereby improve patient survival.

Materials and Methods: We retrospectively analyzed 102 ACLF patients who were hospitalized in the gastroenterology department. The EASL-CLIF criteria were used to define the ACLF. The demographic characteristics and biochemical examination results of the patients were acquired, and seven scores (CTP score, MELD score, MELD-Na, CLIF ACLF score, CLIFC OF score, CLIF SOFA score) were calculated 24 hours after admission. All patients were observed until loss to follow-up, death, or specific follow-up times (28 days, 3 months, 6 months), which were calculated after the initial hospital admission. The receiver operating characteristic (ROC) curve was employed to estimate the power of six scores to forecast ACLF patients' outcome.

Results: All scores were distinctly higher in nonsurviving patients than in surviving patients and had predictive value for outcome in patients with ACLF at all time points $(\mathrm{P}<0.050)$. The areas 
under the ROC curve (AUROCs) of the CLIF-SOFA score were higher than those of other scores at all time points. The comparison of the AUROC of the CLIF-SOFA score with other scores was statistically significant at 28 days $(P<0.050)$, which was the only time point at which it was greater than 0.800 .

Conclusion: Patients with ACLF have high mortality. These six scores are effective tools for assessing the prognosis of ACLF patients. The CLIF-SOFA score is especially effective for evaluating 28-day mortality.

Key word: Acute-on-chronic liver failure; prognosis; scoring model

\section{Introduction}

Acute-on-chronic liver failure (ACLF) is a clinical syndrome characterized by the rapid deterioration of liver function due to acute injury. Patients diagnosed with ACLF often have multiple organ failures and high short-term mortality ${ }^{(1)}$. Patients with chronic liver disease may progress to liver failure induced by enhanced viral replication, combined with bacterial or fungal infection and liver injury due to drug abuse or alcoholism ${ }^{(2)}$. The basic etiology of ACLF is mainly alcoholism in European and American countries; however, hepatitis virus infection is the leading etiology of ACLF in Asian countries, especially in China ${ }^{(3)}$. Although treatments such as liver transplantation and hemodialysis markedly improve survival in the short term, they are not extensively obtainable in clinical practice because of their high costs, the limited availability of liver resources, and the need for hospitalization. ACLF causes a heavy economic burden on patients. ACLF patients perform obvious differences in accordance with morbidity and survival. So, it is essential to develop an applicable prognostic score to estimate the outcomes in ACLF patients and help guide doctors in determining the treatment options according to the predicted outcomes.

Some prognostic scores have been established previously. The Child-Turcotte-Pugh (CTP) score 
was first established as a widely utilized liver-specific score nearly 50 years ago ${ }^{(4)}$. Wiesner's research analyzed data and established the Model for End-Stage Liver Disease (MELD) score; the MELD score is superior to the CTP score with regard to the prediction of 3-month mortality in patients with chronic end-stage liver disease ${ }^{(5)}$. The MELD combined with serum sodium concentration (MELD-Na) score is related to the MELD score and has improved prognostic efficacy in cirrhotic patients awaiting liver transplantation ${ }^{(6)}$. In the EASL-CLIF acute-onchronic liver failure in cirrhosis (CANONIC) study, ACLF was defined using a novel scoring system called the CLIF-sequential organ failure assessment score (CLIF-C SOFA), which is a modification of the original SOFA score. The EASL-CLIF consortium also developed the CLIF consortium organ failure score (CLIF-C OF), which simplified the original CLIF-SOFA. Through further studies, Jalan et al found that age and white blood cell count were independent risk factors for mortality and established the CLIF-C ACLF score ${ }^{(7)}$. The CLIF-C ACLF score not only assesses the effects of extrahepatic organ injury, coagulation and circulatory failure but also includes age and inflammatory indicators; the CLIF-C ACLF score has high clinical value for evaluating the prognosis of ACLF. Up to now, there are less study on comparing all methods for the evaluation and prediction of prognosis in ACLF patients with a variety of etiologies, especially among Asians. Our study was designed to assess the short-term and long-term discriminative power of all of the above scores in ACLF patients to direct clinical practice.

\section{Material and methods}

\section{Study patients}

Our study was a single-center retrospective study that was completed in acute-on-chronic liver failure patients hospitalized in our institute between January 2015 and December 2018. Patients were included when they fulfilled these criteria: (a) $\geq 18$ years old and (b) diagnosed with cirrhosis and ACLF (defined by the EASL-CLIF Consortium). Exclusion criteria included (1) hepatocellular carcinoma, (2) previous liver transplantation, (3) complications with other severe chronic extrahepatic diseases and (4) infection with human immunodeficiency virus. Our study 
109

110

111

112

113

114

115

116

117

118

119

120

121

122

123

was approved by the Ethics Committee of the First Affiliated Hospital of Nanchang University (No. 2015-1203). All the patients signed the informed consent.

\section{Definitions}

Cirrhosis was defined by laboratory tests, radiologic imaging, endoscopy or liver biopsy. The ACLF criteria and organ failures were defined based on the CLIF-SOFA score according to the EASL-CLIF Consortium. The ACLF grading system classifies patients with ACLF in one of 3 grades according to the number of organ failures as per the CLIF-SOFA score as follows: Grade 1 if (1) single kidney failure (serum creatinine level $\geq 2.0 \mathrm{mg} / \mathrm{dl}$ ) or (2) another organ failure (respiration, circulation, coagulation, or liver) is accompanied by grade I-II (West Haven criteria) hepatic encephalopathy (HE) and/or a serum creatinine level of 1.5-1.9 mg/dl, or (3) single cerebral failure (grade III-IV HE) is present with a serum creatinine level of $1.5-1.9 \mathrm{mg} / \mathrm{dl}$; grade 2 if 2 organ failures are identified; or grade 3 if 3 or more organ failures have been diagnosed. The Child-Pugh score was computed based on albumin, ascites, hepatic encephalopathy, prothrombin time $(\mathrm{PT})$, and serum bilirubin ${ }^{(4)}$. The MELD formula was: $3.8 \times \log$ (bilirubin) $+9.6 \times \log ($ creatinine $)+11.2 \times \log ($ INR $)+6.43^{(8)}$. The MELD-Na score was calculated as below: MELD $-\mathrm{Na}=[0.025 \times \mathrm{MELD} \times(140-\mathrm{Na})]+140^{(6)}$. The CLIF-SOFA score was computed as the sum of the scores for six organ systems, including the cardiovascular, hepatic, coagulation, respiratory, nervous, and renal systems ${ }^{(9)}$. The CLIF-C OF score includes the revised six organ systems of the CLIF-SOFA score. The CLIF-C ACLF score was revised according to the CLIFSOFA score and was computed with the formula: $10 \times[0.63 \times \log$ (white-cell count) + $0.33 \times$ CLIF-C OF $+0.04 \times$ age -2$]^{(7)}$.

\section{Study protocols}

Patients with ACLF were included in the study. During hospitalization, data were collected regarding medical records, demographics, the presence of other comorbidities, clinical features, the number of complications and type of decompensation, the etiology of cirrhosis, and blood haematological index at admission (such as blood platelet count, white blood cell count, the INR, 
135

136

137

138

139

140

141

142

143

144

145

146

147

148

149

150

151

152

153

154

155

156

157

158

159

160

161

162

renal function test, liver function test). The patients were followed up for 6 months to obtain survival information. Patients with incomplete follow-up at 28 days, 3 months, and 6 months were not included in the final analysis of the corresponding time.

\section{Statistical analysis}

The statistical analyses were performed using SPSS software version 20.0 (SPSS Inc., Chicago, IL). Continuous variables were expressed as the mean \pm standard deviation (SD) or medians (interquartile range $[\mathrm{IQR}]$ ), and categorical data were expressed as percentages. Differences in variables were analyzed using Student t-tests or the Mann-Whitney U test. Categorical variables are described as the frequencies (percentages [\%]) and were compared with chi-squared or Fisher's exact tests. Receiver operating characteristic (ROC) curves were used to measure the performance of the score for the prediction of 28-day, 3-month, and 6-month mortality in patients with ACLF. The specificity, sensitivity, negative likelihood ratio (NLV) and positive likelihood ratio (PLV) were computed for each cut-off value. The cut-off point was obtained by Youden's index with greatest Sensitivity and Specificity ${ }^{(10)}$. The comparing of the areas under the ROC curve (AUROCs) was performed by Delong-test. 0.050 of two-tailed was significant meaning.

\section{Results}

\section{Characteristics of ACLF patients}

There were 102 patients in this study. During the study period, 92 patients were enrolled in the analysis of the outcomes at 28 days; subsequently, 3 patients were lost to follow-up, and 89 patients were finally enrolled at both 3 and 6 months. The flowchart is shown in Figure 1, and the demographic and biochemical characteristics of the study population are summarized in Table 1. The mean ( \pm standard deviation) age of the 102 patients was $56.96( \pm 12.18)$ years. The leading cause of decompensation events responsible for hospitalization was variceal bleeding (70/102, 68.6\%). The ACLF patient distribution was grade $1(31 / 10230.4 \%)$, grade $2(45 / 102$, $44.1 \%)$, and grade 3 (26/102 25.5\%). The most common degree of ascites was moderate (28/102 $27.5 \%$ ), followed by severe (25/102 24.5\%) and mild (13/102 12.7\%). Forty-nine (48\%) patients had undergone endoscopic hemostasis, 41 (40.2\%) patients had undergone mechanical 
163 ventilation, and $66(64.7 \%)$ patients had used vasopressors. In the 28-day and 3-month analyses, 164 the mean age was $57.5( \pm 12)$ years and $57.8( \pm 12)$ years, respectively, and $62(67.4 \%)$ and 59 $165(66.3 \%)$ patients were male. The leading cause of liver cirrhosis is Hepatitis virus infection and 166 variceal bleeding accounts for the majority of hospitalizations. The distributions of patients who 167 were included in the complete follow-up within 28 days and were included in the complete 168 follow-up within 3 months were similar to that of all 102 patients in terms of ascites grade, 169 ACLF grade, and treatment strategy. A total of 47 (46.1\%), 58 (56.9\%), and 61 (59.8\%) patients 170 died within 28 days, 3 months, and 6 months, respectively. The causes of death at 6 months were as follows: 3 (4.9\%) patients had cardiogenic shock, $6(9.8 \%)$ patients had infectious shock, 12 (19.7\%) patients had respiratory failure, 18 (29.5\%) patients had hemorrhagic shock, 19 (31.1\%) patients had liver-related complications (4 patients had liver failure, 15 patients had hepatic encephalopathy) and 3 (4.9\%) patients had an uncertain cause of death. The causes of death at 28 days, 3 months, and 6 months are outlined in Supplement Table 1.

Comparison of prognostic scores between the nonsurviving group and the surviving patients

The comparison of the six scores of patients with ACLF were shown in Table 2. ACLF patients were grouped into surviving and nonsurviving groups based on their 28-day, 3-month, and 6month outcomes. The non-surviving patients had a higher CTP score, MELD score, CLIF-C OF score, CLIF-SOFA score and CLIF-ACLF score, compared with surviving patients $(\mathrm{P}<0.050)$. Although the comparison of the MELD-Na score was not statistically significant $(P=0.081)$, it was still higher in the nonsurviving group. Statistically significant differences were found for the CTP score, MELD-Na score, MELD score, CLIF-SOFA score, CLIF-ACLF score, CLIF-C OF score at 3 months and 6 months $(P<0.050)$.

\section{Predictive ability for 28-day, 3-month and 6-month outcome in ACLF patients.}

The discriminative ability of the CTP score, MELD score, MELD-Na score, CLIF-C OF score, and CLIF-ACLF score calculated for 28-day, 3-month, and 6-month survival is summarized in Table 3. At 28 days, the CLIF SOFA score had the highest AUROC (0.805, 95\%CI:0.715-0.896), followed by the CLIF-ACLF score (0.741, 95\%CI: 0.640-0.843), CLIF-C OF score (0.712, 95\%CI: 0.676 to 0.869$)$, CTP score $(0.707,95 \% \mathrm{CI}: 0.600-0.813)$, MELD score $(0.673$, 95\% CI: 
193

194

195

196

197

198

199

200

201

202

203

204

205

206

207

208

209

210

211

212

213

214

215

216

217

218

0.560-0.787), and MELD-Na score (0.606, 95\%CI: 0.487 to 0.724$)$. When predicting 3-month and 6-month mortality, the CLIF-C SOFA score both had the highest AUROC $(0.751,95 \% \mathrm{CI}$ : 0.646-0.857; $0.742,95 \% \mathrm{CI}: 0.633-0.852$, respectively), by contrast, CTP score both had the lowest AUROC $(0.641,95 \% \mathrm{CI}$ : $0.521-0.760 ; 0.640,95 \% \mathrm{CI}: 0.518-0.762$, respectively). The ROC curves for the prognostic scores are shown in Figure 2. All prognostic scores were able to predict mortality at 28 days, 3 months, and 6 months $(\mathrm{P}<0.050)$.

\section{Comparing the predictive performance of all scores}

As shown in Table 3, the AUROC of the CLIF-SOFA score is superior to those of the other five scores with regard to 28-day, 3-month, and 6-month mortality. The CLIF-SOFA has the highest predicting value in 28-day mortality with the AUROC of 0.805 . The predicting performer of CLIF-SOFA is significantly higher than CTP score, MELD-Na score, MELD score, CLIF-C OF score, and CLIF-ACLF score $(P<0.050)$. At 3 months and 6 months, the comparison of AUROCs between the CTP score and the CLIF-SOFA score was statistically significant $(P<0.050)$; however, the comparisons of AUROCs between the CLIF-C OF score, CLIF-ACLF score, MELD-Na score and MELD score were not significant $(P>0.050)$. At 28 days, the AUROC of MELD-Na was lower than other five scores.

\section{Discussion}

It is important to develop predictive scores that can identify patients who are at high risk of mortality, enabling the early provision of effective treatment to reduce mortality, especially in diseases with high mortality rates. ACLF is a clinical syndrome with a high mortality rate that is characterized by the development of acute decompensation (encephalopathy, ascites, gastrointestinal hemorrhage) and organ failure (such as kidney, renal, hepatic, coagulation, respiration and circulation), so prognostic assessment is an indispensable for ACLF patients ${ }^{(9)}$. However, in the clinical setting, the prognosis is often hard to predict for certain patients because of different factors, such as etiology, disease stage, and complications. Previous studies have shown that many different scores have predictive value for mortality in ACLF patients. It is very 
219 important to choose the most efficient score for predicting mortality in Asian patients in clinical 220 treatment. The clinical characteristics of ACLF patients in Asian is completely different form 221 patients in Europe and America. In this study, the leading etiology of liver cirrhosis was hepatitis

222 virus infection (58.8\%), followed by alcohol-related cirrhosis (34.1\%), which was similar to the primary etiologies of liver disease in most Asian countries.

It is not surprising that the mortality of ACLF patients was high in this study, as that it consistent with previous research ${ }^{(11-13)}$. The mortality rate was $46.1 \%$ in the short term (28 days), and the mortality rate was $59.8 \%$ in the long term (6 months). The high mortality rate, which we find appalling, has spurred us to meaningfully contribute. Effective and inexpensive treatment strategies for patients with low socioeconomic status are limited because of the high costs associated with liver transplant and hemodialysis, partially in developing countries. The economical load produced by ACLF is still severe. Predicting the prognosis of patients with ACLF may be more important than treatment from the perspective of health economics for lowincome families.

Recently, the CLIF-ACLF score, CLIF-C OF score, CLIF-SOFA score have been used to evaluate prognosis in ACLF patients ${ }^{(14,15)}$. To the best of our knowledge, although the discriminative ability of these scores for predicting outcomes in ACLF patients has been illustrated, different conclusions have been drawn regarding the relative predictive value of these scores because of differences in study populations or observation durations.

The predictive value of the six scores (CTP score, MELD score, MELD-Na, CLIF-ACLF score, CLIF-C OF score, and CLIF-SOFA score) was compared at 28 days, 3 months, and 6 months. The AUROC of CLIF-SOFA is higher than other prognostic scores at 28 days, 3 months, and 6 months in our cohort, especially at 28 days. The CLIF-SOFA score provides a comprehensive and effective assessment of the severity of organ failure in ACLF patients and takes into account multiple systems, including the hepatic, renal, coagulation, respiratory, circulatory and nervous systems; it was established by the European Liver Disease Collaboration Group for Liver Failure 
245 in 2013. Sy E's study indicated that the predictive value of the CLIF-SOFA score is better than 246 those of the CTP score and MELD score for short-term outcomes ${ }^{(16)}$. Any score has its 247 advantages and disadvantages. Although the predictive value of the CLIF-SOFA score is high, 248 the calculation of the CLIF-SOFA score is complicated due to the inclusion of more indicators. 249 The Child-Pugh score is computed based on the prothrombin time, ascites, serum bilirubin, 250 albumin, and hepatic encephalopathy ${ }^{(4)}$. The presence or absence of hepatic encephalopathy and 251 ascites, which forms part of the CTP score, is subjective and has no clear cut-off value. The 252 MELD score contains contains three indicators: the INR, creatinine and bilirubin; it is vulnerable

253

254 to confounding by hemorrhaging, ascites and the use of diuretics, with the absence of clearly defined cutoff values for categorizing cirrhotic patients ${ }^{(17)}$. The occurrence of hyponatremia is closely related to the prognosis of patients with cirrhosis, particularly patients with ascites; therefore, the MELD-Na score has been created based on the MELD score ${ }^{(18)}$. However, the MELD score had a lower AUROC than the other five scores at all time points in this study. This may be due to the main complications of patients in this study. The patients were mainly enrolled from the Department of Gastroenterology and needed endoscopic treatment for bleeding esophageal gastric varices $(70 / 102,68.6 \%)$. The number of cirrhosis patients with ascites as the primary reason for hospitalization was very small (6/102 5.9\%), Previous study have confirmed the ascites is the main complication of liver cirrhosis ${ }^{(19)}$, and ascites is associated with a high risk of developing further complications of cirrhosis such as dilutional hyponatremia ${ }^{(20)}$, Because of the number of patients with ascites are small, so the MELD-Na score may not play an important role in predicting patients' mortality. which may explain why the discriminative power of the MELD-Na score is lower than other five scores. The predicting value of the CTP, MELD-Na, and MELD scores in ACLF is not completely prefect because indicators reflecting systemic inflammation and organ failure is lacking. The CANONIC study had shown the advantage of the CLIF-ACLF, CLIF-SOFA, and CLIF-C OF scores over the CTP, MELD-Na, and MELD scores for the prediction of mortality in ACLF patients, which is according with the results in our study $^{(7)}$. Jalan et al. first proposed the CLIF-C OF score in 2014 and proved that the value of the 
272 CLIF-C OF score is equivalent to that of the CLIF-SOFA score for the prediction of mortality ${ }^{(7)}$.

273 Considering the effects of white blood cell (WBC) count and age on prognosis, Jalan et al 274 established the CLIF-ACLF score based on the CLIF-C OF score ${ }^{(21)}$. The CLIF-ACLF score not 275 only considers the effects of extrahepatic organ damage, coagulation and circulatory system 276 failure on the prognosis but also includes the WBC count, which reflects the severity of 277 inflammation; the CLIF-ACLF score was superior to the CTP, MELD-Na, and MELD scores ${ }^{(21)}$. 278 Despite the high predictive value of the CLIF-ACLF score and CLIF-C OF, these scores were established based on patients from European countries and the US with alcohol-related liver disease, and further researches are needed to explore whether they are applicable to Asian populations. Our research results have indicated that the scores also apply to Asian populations.

Several limitations existed in this study. First, this was a retrospective study, the number of patients included in our study was still not large, and some patients were lost to follow-up, which may have resulted in selection bias. Second, the scores were evaluated when admission to hospital and did not reflect the dynamic changes. Finally, the leading etiologies in patients in our study were hepatitis B virus infection, but most of the patients were diagnosed according to the EASL-ACLF criteria, leading to etiological bias.

In conclusion, our data reveal that the CTP score, MELD score, MELD-Na, CLIF-C OF score, CLIF-SOFA score, CLIF-ACLF score are effective tools for predicting the prognosis in ACLF patients. The CLIF-SOFA score has better discriminative power for the evaluation of short-term mortality, and may help improve the management of ACLF patients.

\section{Figure 1 The flowchart in our study}

Figure 2 ROC for the MELD-Na score, MELD score, Child-Pugh score, CLIF-C OF score, CLIF-SOFA score and CLIF-ACLF score for predicting mortality at 28 days, 3 months and 6 
297 months. MELD: the model for end-stage liver disease score; Child-Pugh: the Child-Pugh score.

\section{Acknowledgments}

We would like to thank the National Natural Science Foundation of China and the "Gan-Po Talent 555" Project of Jiangxi Province for financial support.

Conflict of Interest statement: The authors declare that there are no conflicts of interest.

\section{Reference}

1. Bernal W, Jalan R, Quaglia A, Simpson K, Wendon J, Burroughs A. Acute-on-chronic liver failure. Lancet. 2015;386(10003):1576-87. on-chronic liver failure: prevalence, characteristics and impact on prognosis. Gut. 2018;67(10):1870-80.

3. Zhao RH, Shi Y, Zhao H, Wu W, Sheng JF. Acute-on-chronic liver failure in chronic

314 hepatitis B: an update. Expert review of gastroenterology \& hepatology. 2018;12(4):341-50. 
316 oesophagus for bleeding oesophageal varices. The British journal of surgery. 1973;60(8):646-9.

317 5. Wiesner R, Edwards E, Freeman R, Harper A, Kim R, Kamath P, Kremers W, Lake J,

318 Howard T, Merion R, Wolfe R, Krom R. Model for end-stage liver disease (MELD) and 319 allocation of donor livers. Gastroenterology. 2003;124(1):91-6.

320 6. Kim WR, Biggins SW, Kremers WK, Wiesner RH, Kamath PS, Benson JT, Edwards E, 321 Therneau TM. Hyponatremia and mortality among patients on the liver-transplant waiting list. 322 The New England journal of medicine. 2008;359(10):1018-26.

323 7. Jalan R, Saliba F, Pavesi M, Amoros A, Moreau R, Gines P, Levesque E, Durand F, Angeli 324 P, Caraceni P, Hopf C, Alessandria C, Rodriguez E, Solis-Munoz P, Laleman W, Trebicka J, 325 Zeuzem S, Gustot T, Mookerjee R, Elkrief L, Soriano G, Cordoba J, Morando F, Gerbes A, 326 Agarwal B, Samuel D, Bernardi M, Arroyo V, Consortium CsiotE-C. Development and 327 validation of a prognostic score to predict mortality in patients with acute-on-chronic liver failure. 328 Journal of hepatology. 2014;61(5):1038-47. G, Dickson ER, Kim WR. A model to predict survival in patients with end-stage liver disease. Hepatology. 2001;33(2):464-70.

9. Moreau R, Jalan R, Gines P, Pavesi M, Angeli P, Cordoba J, Durand F, Gustot T, Saliba F,

Domenicali M, Gerbes A, Wendon J, Alessandria C, Laleman W, Zeuzem S, Trebicka J, 
335 10. YOUDEN WJ. Index for rating diagnostic tests. CANCER-AM CANCER SOC 1950, 3(1):

$336 \quad 32-35$.

337 11. Hernaez R, Kramer JR, Liu Y, Tansel A, Natarajan Y, Hussain KB, Gines P, Sola E, Moreau

338 R, Gerbes A, EI-Serag H, Kanwal F. Prevalence and short-term mortality of acute-on-chronic

339 liver failure: A national cohort study from the USA. Journal of hepatology. 2019;70(4):639-47.

340 12. Mahmud N, Kaplan DE, Taddei TH, Goldberg DS. Incidence and Mortality of Acute-on-

341 Chronic Liver Failure Using Two Definitions in Patients with Compensated Cirrhosis.

342 Hepatology. 2019;69(5):2150-63.

343 13. Sundaram V, Jalan R, Wu T, Volk ML, Asrani SK, Klein AS, Wong RJ. Factors Associated

344 with Survival of Patients With Severe Acute-On-Chronic Liver Failure Before and After Liver

345 Transplantation. Gastroenterology. 2019;156(5):1381-91 e3.

346

14. Engelmann C, Thomsen KL, Zakeri N, Sheikh M, Agarwal B, Jalan R, Mookerjee RP. Validation of CLIF-C ACLF score to define a threshold for futility of intensive care support for patients with acute-on-chronic liver failure. Critical care. 2018;22(1):254.

15. Song DS, Kim TY, Kim DJ, Kim HY, Sinn DH, Yoon EL, Kim CW, Jung YK, Suk KT, Lee SS, Lee CH, Kim TH, Choe WH, Yim HJ, Kim SE, Baik SK, Jang JY, Kim HS, Kim SG, Yang JM, Sohn JH, Choi EH, Cho HC, Jeong SW, Kim MY, Korean Acute-on-Chronic Liver Failure Study G. Validation of prognostic scores to predict short-term mortality in patients with acuteon-chronic liver failure. Journal of gastroenterology and hepatology. 2018;33(4):900-9. 
354 16. Sy E, Ronco JJ, Searle R, Karvellas CJ. Prognostication of critically ill patients with acute355 on-chronic liver failure using the Chronic Liver Failure-Sequential Organ Failure Assessment: A 356 Canadian retrospective study. Journal of critical care. 2016;36:234-9.

357 17. Cholongitas E, Papatheodoridis GV, Vangeli M, Terreni N, Patch D, Burroughs AK. 358 Systematic review: The model for end-stage liver disease--should it replace Child-Pugh's 359 classification for assessing prognosis in cirrhosis? Alimentary pharmacology \& therapeutics. $360 \quad 2005 ; 22(11-12): 1079-89$.

361 18. Biggins SW, Kim WR, Terrault NA, Saab S, Balan V, Schiano T, Benson J, Therneau T, 362 Kremers W, Wiesner R, Kamath P, Klintmalm G. Evidence-based incorporation of serum 363 sodium concentration into MELD. Gastroenterology. 2006;130(6):1652-60.

364 19. De Vusser P, Bounameaux H, Verhaeghe R, De Groote J. Liver cirrhosis, ascites, and 365 peripheral vascular resistance. Lancet. 1985;2(8463):1078. international. 2018;12(Suppl 1):122-34. 2017;66(3):541-53. 


\section{Figure 1}

\section{figure(revised)}

\begin{tabular}{|c|c|}
\hline $\begin{array}{l}500 \text { patients with decompensated cirrhosis } \\
\text { between } 11 / 2013 \text { to } 08 / 2019\end{array}$ & \\
\hline & $\begin{array}{l}398 \text { patients were exclude for the following reasons: } \\
1 \text { patients had HIV infection } \\
393 \text { patients did not meet EASL ACLF criteria at admission } \\
4 \text { patients complicated with other severe chronic extrahepatic } \\
\text { diseases }\end{array}$ \\
\hline \multicolumn{2}{|l|}{102 patients with ACLF at admission } \\
\hline & $\begin{array}{l}4 \text { patients were exclude for loss of follow up within } 28 \text { days } \\
5 \text { patients were exclude for absence of test results } \\
1 \text { were exclude for death less than } 24 \text { hours }\end{array}$ \\
\hline \multicolumn{2}{|l|}{$\begin{array}{l}92 \text { patients were included in the complete } \\
\text { follow-up within } 28 \text { days }\end{array}$} \\
\hline & $\begin{array}{l}3 \text { patients were exclude for loss of follow up between } 28 \text { days } \\
\text { and } 3 \text { months }\end{array}$ \\
\hline \multicolumn{2}{|l|}{$\begin{array}{l}89 \text { patients were included in the complete } \\
\text { follow-up within } 3 \text { months }\end{array}$} \\
\hline & $\begin{array}{l}\text { No patients were loss of follow up between } 3 \text { months and } 6 \\
\text { months }\end{array}$ \\
\hline
\end{tabular}

89 patients were included in the complete follow-up within 6 months 
Figure 2

Figure 2

Receiver operating characteristic curves for the MELD-Na score, MELD score, Child-Pugh score, CLIF-C OF score, CLIF-SOFA score and CLIF-ACLF score for predicting mortality at 28 days, 3 months and 6 months. MELD: the model for end-stage liver disease score; ChildPugh: the Child-Pugh score.
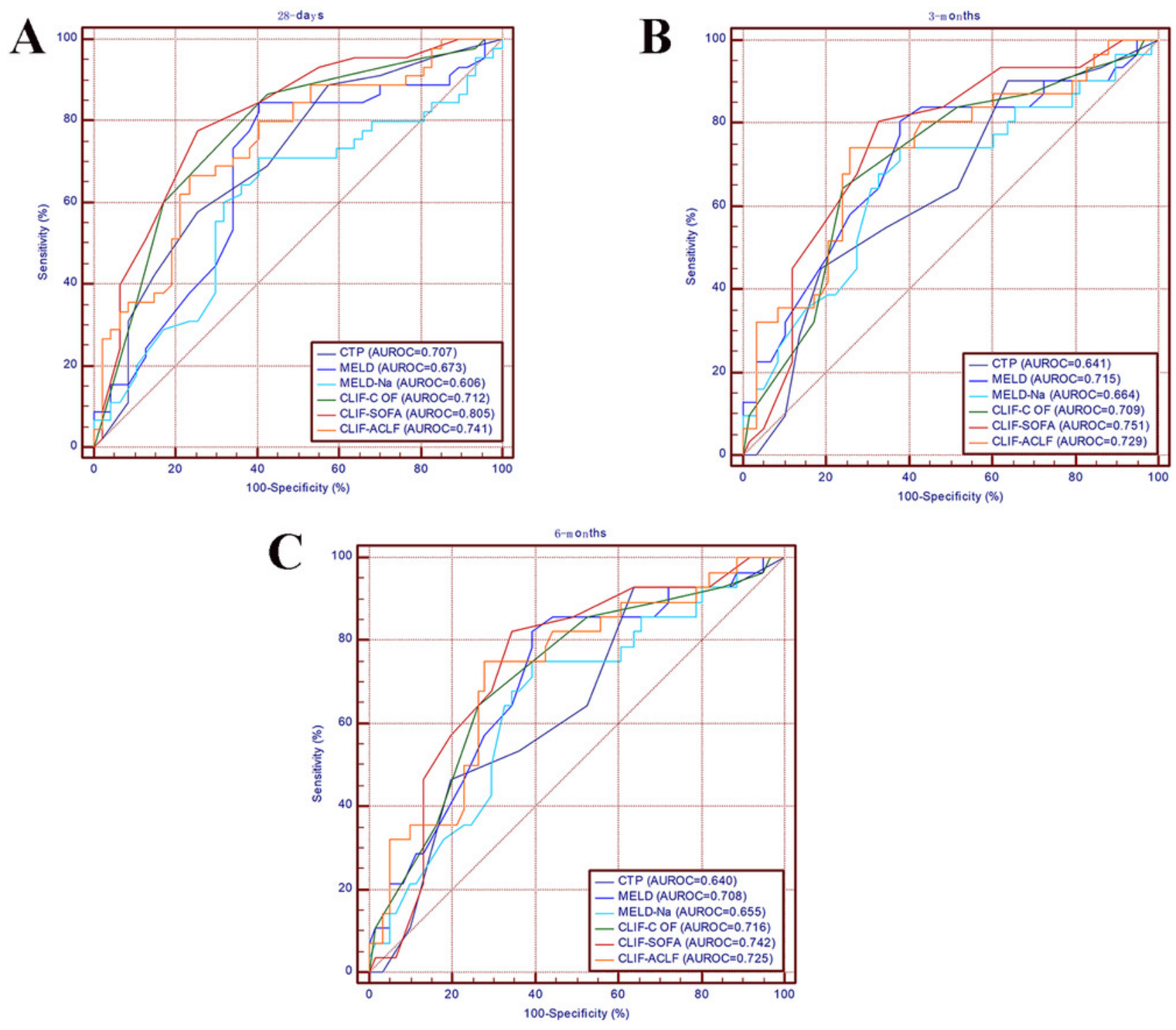


\section{Table 1 (on next page)}

Table 1

Characteristics of Patients in the ACLF cohort 


\begin{tabular}{|c|c|c|c|}
\hline & $\begin{array}{l}\text { Patients with ACLF at admission } \\
\qquad(\mathrm{n}=102)\end{array}$ & $\begin{array}{l}\text { Patients in complete follow-up } \\
\text { within 28-days(n=92) }\end{array}$ & $\begin{array}{l}\text { Patients in complete follow-up within } \\
\text { 3-months or 6-months ( } \mathrm{n}=89)\end{array}$ \\
\hline Age, mean \pm SD & $56.96 \pm 12.18$ & $57.5 \pm 12$ & $57.8 \pm 12$ \\
\hline Sex (male), n (\%) & $70(68.6 \%)$ & $62(67.4 \%)$ & $59(66.3 \%)$ \\
\hline Hospitalization days, median (IQR) & $4(1-11)$ & $4.5(1.25-11.0)$ & $5.0(1.0-11.0)$ \\
\hline \multicolumn{4}{|l|}{ Aetiology of chronic liver disease, $\mathrm{n}(\%)$} \\
\hline Hepatitis B Virus & $59(58.8 \%)$ & $52(57.6 \%)$ & $50(56.1 \%)$ \\
\hline Alcoholic liver disease & $35(34.1 \%)$ & $32(34.7 \%)$ & $31(34.8 \%)$ \\
\hline Hepatitis C Virus & $2(1.9 \%)$ & $2(2.1 \%)$ & $2(2.2 \%)$ \\
\hline Primary biliary cirrhosis & $4(3.9 \%)$ & $4(4.3 \%)$ & $4(4.5 \%)$ \\
\hline Others & $17(16.7 \%)$ & $15(16.3 \%)$ & $15(16.8 \%)$ \\
\hline \multicolumn{4}{|c|}{ Primary reason for hospitalization, $\mathrm{n}(\%)$} \\
\hline Variceal bleeding & $70(68.6 \%)$ & $65(70.7 \%)$ & $62(69.6 \%)$ \\
\hline Ascites & $6(5.9 \%)$ & $5(5.4 \%)$ & $0(5.6 \%)$ \\
\hline Hepatic encephalopathy & $14(13.7 \%)$ & $13(14.1 \%)$ & $13(14.6 \%)$ \\
\hline Infection & $11(10.8 \%)$ & $8(8.7 \%)$ & $8(8.9 \%)$ \\
\hline Others & $1(0.9 \%)$ & $1(1.1 \%)$ & $1(1.1 \%)$ \\
\hline \multicolumn{4}{|l|}{ ACLF grade, $n(\%)$} \\
\hline ACLF grade 1 & $31(30.4 \%)$ & $29(31.5 \%)$ & $28(31.5 \%)$ \\
\hline ACLF grade 2 & $45(44.1 \%)$ & $39(42.4 \%)$ & $37(41.6 \%)$ \\
\hline ACLF grade 3 & $26(25.5 \%)$ & $24(26.1 \%)$ & $24(26.9 \%)$ \\
\hline Endoscopic hemostasis, n (\%) & $49(48 \%)$ & $48(52.2 \%)$ & $46(51.7 \%)$ \\
\hline The degree of ascites, $n(\%)$ & & & \\
\hline
\end{tabular}




\begin{tabular}{|c|c|c|c|c|}
\hline Mild & $13(12.7 \%)$ & $11(12.0 \%)$ & $11(12.3 \%)$ & Table \\
\hline & & & & Characteristic \\
\hline Moderate & $28(27.5 \%)$ & $27(29.3 \%)$ & $25(28.1 \%)$ & $\mathrm{s}$ of Patients \\
\hline Severe & $25(24.5 \%)$ & $24(26.1 \%)$ & $24(26.9 \%)$ & in the ACLF \\
\hline & & & & cohort \\
\hline Hepatocellular carcinoma, $\mathrm{n}(\%)$ & $10(9.8 \%)$ & $10(10.9 \%)$ & $9(10.1 \%)$ & \\
\hline & & & & ACLF: Acute- \\
\hline Mechanical ventilation, $\mathrm{n}(\%)$ & $41(40.2 \%)$ & $37(40.2 \%)$ & $37(41.6 \%)$ & on-chronic liver \\
\hline Vasopressor use, n (\%) & $66(64.7 \%)$ & $60(65.2 \%)$ & $58(65.2 \%)$ & failure; \\
\hline & & & & Standard \\
\hline
\end{tabular}

10 Deviation; IQR: interquartile range;

11 
Table 2 (on next page)

Table 2

The comparison of prognostic scores 


\begin{tabular}{|c|c|c|c|c|c|c|c|c|c|c|}
\hline & & & 28-days & & & 3-months & & & 6-months & \\
\hline & & survivors $(\mathrm{n}=45)$ & non-survivors(n=47) & P-value & survivors $(\mathrm{n}=31)$ & non-survivors(n=58) & P-value & survivors(n=28) & non-survivors $(\mathrm{n}=61)$ & P-value \\
\hline CTP score & $11(9-13)$ & $10(8-12)$ & $12(10-14)$ & 0.001 & $10(8-12)$ & $11.00(10.00-13.25)$ & 0.028 & $10(8-12)$ & $11(10-13.5)$ & 0.033 \\
\hline MELD score & $18(14-25.75)$ & $16(13.5-20)$ & $24(15-29)$ & 0.004 & $15(12-18)$ & $23(15-29)$ & 0.001 & $15(12-18)$ & $23(15-29)$ & 0.002 \\
\hline MELD-Na score & $20.69(15.00-29.00)$ & $18.00(14.00-27.36)$ & $24.00(15.48-29.64)$ & 0.081 & $16.54(13-26.13)$ & $23.27(16-29.67)$ & 0.011 & $17.27(14.00-24.73)$ & $23.00(15.74-29.70)$ & 0.020 \\
\hline CLIF-C OF score & $10(9-11)$ & $9(8-10)$ & $11(10-12)$ & $<0.001$ & $9(8-10)$ & $10.00(9.75-12.00)$ & 0.001 & $9(8-10)$ & $10(9-12)$ & 0.001 \\
\hline CLIF-SOFA score & $10(8-13)$ & $8(6.5-10)$ & $12(10-14)$ & $<0.001$ & $8.55 \pm 2.69$ & $11.46 \pm 3.36$ & $<0.001$ & $8.53 \pm 2.67$ & $11.33 \pm 3.39$ & $<0.001$ \\
\hline CLIF-C ACLF score & $49.59 \pm 10.59$ & $45.01 \pm 9.99$ & $53.98 \pm 9.28$ & $<0.001$ & $44.39 \pm 10.61$ & $52.85 \pm 9.41$ & $<0.001$ & $44.11 \pm 10.36$ & $52.56 \pm 9.66$ & 0.001 \\
\hline
\end{tabular}

2 Table 2. The comparison of prognostic scores

3

4 CTP: Child-Turcotte-Pugh; MELD: model for end-stage liver disease; MELD-Na: model for end-stage liver disease-sodium; CLIF-C OF: chronic liver failure consortium organ function; CLIF-SOFA: chronic

5 liver failure-sequential organ failure assessment; CLIF-C ACLF: chronic liver failure consortium acute-on-chronic liver failure

6

7 


\section{Table 3(on next page)}

Table 3

The efficacy and performance comparison of the prognostic scores for predicting mortality in 28-day,3-month and 6-month 


\begin{tabular}{|c|c|c|c|c|c|c|c|}
\hline CTP score & $0.707(0.600-0.813)$ & $<0.001$ & 10.00 & 57.78 & 74.47 & 2.26 & 0.57 \\
\hline MELD score & $0.673(0.560-0.787)$ & $<0.001$ & 22.00 & 84.44 & 59.57 & 2.09 & 0.26 \\
\hline MELD-Na score & $0.606(0.487-0.724)$ & 0.006 & 22.00 & 71.11 & 59.57 & 1.76 & 0.48 \\
\hline CLIF-SOFA score & $0.805(0.715-0.896)$ & $<0.001$ & 10.00 & 77.78 & 74.47 & 3.05 & 0.29 \\
\hline CLIF-ACLF score & $0.741(0.640-0.843)$ & $<0.001$ & 48.20 & 66.67 & 76.60 & 2.85 & 0.44 \\
\hline CLIF-SOFA score vs CTP & $0.099(0.019-0.179)$ & 0.017 & & & & & \\
\hline CLIF-SOFA score vs MELD & $0.132(0.025-0.240)$ & 0.016 & & & & & \\
\hline CLIF-SOFA score vs CLIF-C OF & $0.054(0.082-0.158)$ & 0.042 & & & & & \\
\hline \multicolumn{8}{|l|}{ 3-months mortality } \\
\hline CTP score & $0.641(0.521-0.760)$ & $<0.001$ & 12.00 & 90.32 & 36.21 & 1.41 & 0.27 \\
\hline MELD score & $0.715(0.598-0.832)$ & $<0.001$ & 19.00 & 80.65 & 62.07 & 2.13 & 0.31 \\
\hline MELD-Na score & $0.664(0.541-0.788)$ & $<0.001$ & 20.52 & 74.19 & 62.07 & 1.96 & 0.41 \\
\hline CLIF-C OF score & $0.709(0.595-0.822)$ & $<0.001$ & 9.00 & 64.52 & 75.86 & 2.67 & 0.47 \\
\hline CLIF-SOFA score & $0.751(0.646-0.857)$ & $<0.001$ & 10.00 & 80.65 & 67.24 & 2.46 & 0.29 \\
\hline CLIF-ACLF score & $0.729(0.615-0.842)$ & $<0.001$ & 48.20 & 74.19 & 74.14 & 2.87 & 0.35 \\
\hline CLIF-SOFA score vs CTP & $0.111(0.016-0.206)$ & 0.023 & & & & & \\
\hline CLIF-SOFA score vs MELD & $0.037(-0.069-0.141)$ & 0.396 & & & & & \\
\hline
\end{tabular}




\begin{tabular}{|c|c|c|c|c|c|c|c|}
\hline CLIF-SOFA score vs MELD-Na & $0.089(-0.023-0.207)$ & 0.126 & & & & & \\
\hline CLIF-SOFA score vs CLIF-C ACLF & $0.043(-0.019-0.106)$ & 0.109 & & & & & \\
\hline CLIF-SOFA score vs CLIF-C OF & $0.023(-0.037-0.113)$ & 0.420 & & & & & \\
\hline \multicolumn{8}{|l|}{ 6-months mortality } \\
\hline CTP score & $0.640(0.518-0.762)$ & $<0.001$ & 12.00 & 92.86 & 36.07 & 1.45 & 0.20 \\
\hline MELD-Na score & $0.655(0.532-0.777)$ & $<0.001$ & 20.52 & 75.00 & 60.66 & 1.91 & 0.41 \\
\hline CLIF-C OF score & $0.716(0.601-0.831)$ & $<0.001$ & 9.00 & 64.29 & 73.77 & 2.45 & 0.48 \\
\hline CLIF-SOFA score & $0.742(0.633-0.852)$ & $<0.001$ & 10.00 & 82.14 & 65.57 & 2.39 & 0.27 \\
\hline CLIF-ACLF score & $0.725(0.610-0.840)$ & $<0.001$ & 48.20 & 75.00 & 72.13 & 2.69 & 0.35 \\
\hline CLIF-SOFA score vs MELD-Na & $0.098(-0.024-0.201)$ & 0.107 & & & & & \\
\hline CLIF-SOFA score vs CLIF-C ACLF & $0.036(-0.023-0.094)$ & 0.210 & & & & & \\
\hline CLIF-SOFA score vs CLIF-C OF & $0.027(-0.053-0.109)$ & 0.406 & & & & & \\
\hline
\end{tabular}

1 Table 3. The efficacy and performance comparison of the prognostic scores for predicting mortality in 28-day,3-month and 6-month 
2 ROC: receiver operating characteristic; PLV: positive likelihood ratio; NLV: negative likelihood ratio; CTP: Child-Turcotte-Pugh; MELD: model for end-stage liver disease;

3 MELD-Na: model for end-stage liver disease-sodium; CLIF-C OF:CLIF consortium organ function; CLIF-SOFA: chronic liver failure-sequential organ failure assessment; CLIF-

4 C ACLF:CLIF consortium acute-on-chronic liver failure; CI: Confidence interval 